Pacific Journal of Mathematics

AN ARTIN RELATION (MOD 2) FOR FINITE GROUP ACTIONS 


\title{
AN ARTIN RELATION (MOD 2) \\ FOR FINITE GROUP ACTIONS ON SPHERES
}

\author{
RoNALD M. DOTZEL
}

\begin{abstract}
Recently it has been shown that whenever a finite group $G$ (not a $p$-group) acts on a homotopy sphere there is no general numerical relation which holds between the various formal dimensions of the fixed sets of $p$-subgroups ( $p$ dividing the order of $G$ ). However, if $G$ is dihedral of order $2 q$ ( $q$ an odd prime power) there is a numerical relation which holds $(\bmod 2)$. In this paper, actions of groups $G$ which are extensions of an odd order $p$-group by a cyclic 2-group are considered and a numerical relation $(\bmod 2)$ is found to be satisfied (for such groups acting on spheres) between the various dimensions of fixed sets of certain subgroups; this relation generalises the classical Artin relation for dihedral actions on spheres.
\end{abstract}

0. Introduction. When a $p$-group $P$ acts on a mod $p$ homology $n$-sphere $X$, the fixed point set, $X^{H}$, of any subgroup $H$ has the $\bmod p$ homology of an $n(H)$-sphere, for some integer $n(H)$. The function from subgroups of $P$ to integers defined by $H \rightarrow n(H)$ is called the dimension function and any such function arising in this way is known to originate in a real representation of $P$ (see [2]). If $P$ is elementary abelian, the Borel identity holds (see [1, pg. 175]):

$$
n-n(P)=\sum(n(H)-n(P))
$$

(sum over all $H \leq P$ such that $P / H=\mathbf{Z}_{p}$ ). The motivation for this identity comes from consideration of representations of $P$.

Now suppose $G$ is the dihedral group $D_{p}$ ( $p$ odd prime) (a semidirect product of $\mathbf{Z}_{p}$ and $\mathbf{Z}_{2}$ via the automorphism of $\mathbf{Z}_{p}, g \rightarrow g^{-1}$ ). If $V$ is a real representation of $G$, one can by considering the real irreducible representations of $G$, write down the following Artin relation,

$$
\operatorname{dim} V^{G}=\operatorname{dim} V^{\mathbf{Z}_{2}}-\left(\frac{\operatorname{dim} V-\operatorname{dim} V^{\mathbf{Z}_{p}}}{2}\right) .
$$

In [3], K. H. Dovermann and Ted Petrie show that for actions of $D_{p}$ (and more generally any non $p$-group) on a homotopy sphere one cannot expect to find a numerical relation between the various dimensions of the fixed sets (in particular for smooth actions of $D_{p}$ one cannot expect the Artin relation to hold). However, in [8, Thm. 1.3], E. Straume has shown that 
the Artin relation does hold, $(\bmod 2)$. Specifically,

TheOREM ([8, Thm. 1.3.]): If $X$ is a mod $2 p$ homology $n$-sphere (i.e., $\left.X \sim_{2 p} S^{n}\right)$ with an action of $D_{p}=G$ and $X^{\mathbf{Z}_{p}} \sim_{p} S^{l}, X^{\mathbf{Z}_{2}} \sim_{2} S^{m}$ then $\chi\left(X^{G}\right)=\chi\left(S^{d}\right)$ where

$$
d \equiv m-\left(\frac{n-l}{2}\right) \quad(\bmod 2)
$$

In this paper we will generalize the Straume's result, and hence the Artin relation, considerably. Suppose $G$ is a finite group which is an extension of an odd order $p$-group $P$ by a cyclic 2-group $Q=\mathbf{Z}_{2^{k}}$; $P \rightarrow G \rightarrow Q$. We will call such groups $G$, " $p$-elementary", though this is not quite standared. Such $G$ are always semi-direct products (SchurZassenhaus Lemma) via a homomorphism $\mathbf{Z}_{2^{k}} \stackrel{\phi}{\rightarrow} \operatorname{Aut}(P)$. If $G$ acts on $X \sim_{2 p} S^{n}$, we have:

THEOREM 1. There exists a sequence of subgroups $e=P_{m} \triangleleft P_{m-1} \triangleleft$ $\cdots \triangleleft P_{1} \triangleleft P_{0}=P$ and a corresponding sequence of non-negative integers $k_{1} \leq k_{2} \leq \cdots \leq k_{m}$ such that $\chi\left(X^{G}\right)=\chi\left(S^{d}\right)$ where

$$
d \equiv n\left(\mathbf{Z}_{2^{k}}\right)-\left(\sum_{i=1}^{m} \frac{n\left(P_{l}\right)-n\left(P_{i-1}\right)}{2^{k-k_{l}}}\right) \quad(\bmod 2) .
$$

It should be noted here that the sequence of subgroups can be selected so that each factor group $P_{i-1} / P_{i}$ is an irreducible representation of $\mathbf{Z}_{2^{k}}$ over the field $\mathbf{Z}_{p}$. If this is done, then by a Jordan-Hölder type theorem the length $m$ is unique. Also, the subgroups $P_{i}$ and the integers $k_{i}$ depend entirely on the group structure of $G$. It isn't difficult to verify that a $p$-group with an action of $\mathbf{Z}_{2^{k}}$ has a decomposition similar to the above; we have taken pains in Lemma 2 below to ensure that one exists of an especially nice type. Also, we should regard Theorem 1 as a generalization of the situation for linear representations (see the remark following §3).

I would like to express sincere thanks to the referee, whose comments resulted in substantial improvements.

1. Irreducible representations of $Z_{2^{k}}$ over $Z_{p}$ ( $p$ odd prime). In this section we want to determine the irreducible representations of $\mathbf{Z}_{2^{k}}$ over $\mathbf{Z}_{p}$. The necessary results are contained in Lemma 1.

From now on the cyclic group $\mathbf{Z}_{2}$, will be written $C(j)$. If $\lambda$ is a $2^{j}$ root of -1 in $\mathbf{Z}_{p}, \mathbf{Z}_{p}^{\lambda}$ denotes the one-dimensional representation of $C(j+1)$ given by multiplication by $\lambda$ (this includes the case $\lambda=-1$, corresponding to $j=0$ and $C(1)$, in which case we write $\mathbf{Z}_{p}^{-}$). 
For any $m$ such that $1 \leq m \leq k$, one can consider the induced representation of $C(m)$ over $\mathbf{Z}_{p}$, Ind $\operatorname{In}_{C(1)}^{C(m)}\left(\mathbf{Z}_{p}^{-}\right)$, which we write as $\rho_{m}$. As a vector space over $\mathbf{Z}_{p}, \rho_{m}$ has dimension $2^{m-1}$ and a generator of $C(m)$ acts on a basis $\left\{a_{i}\right\}_{i=1}^{2^{m-1}}$ by $a_{l} \rightarrow a_{l+1}$ if $i<2^{m-1}$ while $a_{2^{m-1}} \rightarrow-a_{1}$. In general, if $G$ is any group, $H \leq K \leq G$ are subgroups and $V$ is a representation of $H$ over some field, then induction is transitive, i.e. $\operatorname{Ind}_{H}^{G}(V)=$ $\operatorname{Ind}_{K}^{G}\left(\operatorname{Ind}_{H}^{K}(V)\right)$. Also if $V=V_{1}+V_{2}$ then $\operatorname{Ind}_{H}^{G}(V)=\operatorname{Ind}_{H}^{G}\left(V_{1}\right)+$ Ind $_{H}^{G}\left(V_{2}\right)$. (For more information on induced representations, see [7; Chapter 7]).

Now there is a one-to-one (up to similarity) correspondence between faithful irreducible representations of $C(k)$ over $\mathbf{Z}_{p}$ and the irreducible factors of $x^{2^{k-1+1}}$ (consider the characteristic polynomial of a generator of $C(k)$ ). Our main concern here, therefore, will be to understand the factorisation of $x^{2^{k-1+1}}$ over $\mathbf{Z}_{p}$. Given any irreducible factor of $x^{2^{k-1+1}}$, note that if $\alpha$ is a root then the companion matrix in $\mathbf{Z}_{p}(\alpha)$ provides a representation of $C(k)$ which is faithful, irreducible, and such that the generator of $C(k)$ has the given factor as its characteristic polynomial. In the following lemma, evidently (a) is well-known - a proof is included for completeness.

LEMMA 1. (a) The irreducible factors of $x^{2^{k-1+1}}$ all have the same degree $d$ and that degree is the order of $p\left(\bmod 2^{k}\right)$, i.e. $p^{d} \equiv 1\left(\bmod 2^{k}\right)$.

(b) If $k=1$ or if $k>1$ and $p \equiv 1(\bmod 4)$ then all the irreducible, faithful representations of $C(k)$ over $\mathbf{Z}_{p}$ are either 1-dimensional or are induced up from a 1-dimensional representation of a proper subgroup, all of the same $\operatorname{dim}=2^{k-l}$.

(c) If $k>1$ and $p \equiv 3(\bmod 4)$ then all the faithful irreducible representations of $C(k)$ over $\mathbf{Z}_{p}$ are either 2-dimensional or are induced up from a 2-dimensional representation of a proper subgroup, all of the same $\operatorname{dim}=$ $2^{k-1+1}$.

Proof. (a) Let $g(x)$ be an irreducible factor of degree $d$ of $x^{2^{k-1}}+1$. Then $g(x)$ is the minimal polynomial for a primitive $2^{k}$ root of 1 , say $\alpha$. Consider the splitting field of $x^{p^{d}}-x$, which is just $\mathbf{Z}_{p}(\alpha)$ (since the degree of $g$ is $d$ ). Thus $\alpha^{p^{d}-1}=1$, so that $2^{k} \mid p^{d}-1$ (since $\alpha$ is a primitive root of 1). Now let $\hat{d}$ be any natural number such that $2^{k} \mid p^{\hat{d}}-1$. We claim that $d \leq \hat{d}$, establishing (a). Let $F$ be the splitting field of $x^{p^{\hat{d}}}-x$ and let $\phi: F \rightarrow F$ be the generator of the Galois group over $\mathbf{Z}_{p}$ given by $y \rightarrow y^{p}$. Suppose $\alpha, \phi(\alpha), \ldots, \phi^{n}(\alpha)$ are all distinct where $1 \leq n \leq \hat{d}-1$ and consider the polynomial $h(x)=\prod_{i=0}^{n}\left(x-\phi^{i}(\alpha)\right)$. The coefficients are symmetric functions in the $\phi^{i}(\alpha)$ and are fixed by $\phi$ hence belong to $\mathbf{Z}_{p}$. 
Since $h(\alpha)=0$, it follows that $d \leq n+1 \leq \hat{d}$. Thus $d=$ degree of $g$ is the order of $p\left(\bmod 2^{k}\right)$.

(b) If $k=1$, the only faithful, irreducible representation of $C(1)$ is $\mathbf{Z}_{p}^{-}$. So, we will assume that $k>1$ and that $p \equiv 1(\bmod 4)$. Let $l$ be the largest integer such that $p \equiv 1\left(\bmod 2^{l}\right)$. If $k \leq l$, then $p \equiv 1\left(\bmod 2^{k}\right)$ and part (a) implies that any faithful irreducible representation of $C(k)$ has dimension 1 , and these are given by multiplication by a $2^{k-1}$ root of $-1, \lambda$. These are the representations $\mathbf{Z}_{p}^{\lambda}$. If $k>l$ and $f(x)$ is an irreducible factor of $x^{2^{l-1}}+1(\operatorname{deg} f$ is 1 , say $f(x)=x-\lambda)$ then $g(x)=f\left(x^{2^{k-1}}\right)$ has degree the order of $p\left(\bmod 2^{k}\right)$, is a factor of $x^{2^{k-1}}+1$ and is irreducible. On the other hand the characteristic polynomial of $\operatorname{Ind}_{C(l)}^{C(k)}\left(\mathbf{Z}_{p}^{\lambda}\right)$ is $x^{2^{k-1}}-\lambda$ (note that this representation has dimension $2^{k-l}$ ).

(c) If $k>1$ and $p \equiv 3(\bmod 4)$, let $l$ be the largest integer such that $p \equiv-1\left(\bmod 2^{l-1}\right)$. If $k \leq l$ then $p \equiv-1\left(\bmod 2^{k-1}\right)$ and $p^{2} \equiv 1\left(\bmod 2^{k}\right)$. Thus any irreducible factor of $x^{2^{k-1}}+1$ has degree 2 and so the dimension of the corresponding representation is 2. If $k>l$ and $f(x)$ is an irreducible factor of $x^{2^{l-1}}+1$ (of degree 2) then $g(x)=f\left(x^{2^{k-1}}\right)$ has degree the order of $p\left(\bmod 2^{k}\right)$, is a factor of $x^{2^{k-1}}+1$ and is irreducible. However, the characteristic polynomial of $\operatorname{Ind}_{C(l)}^{C(k)}(V)$ is $g(x)$ where $V$ is a two-dimensional representation corresponding to $f(x)$ (note that this representation has dimension $\left.2^{k-l+1}\right)$. This completes the proof of the lemma.

2. Normal chief series for $p$-elementary groups. A normal chief series for a $p$-group $P$ is a normal series whose adjacent quotients are elementary abelian. When $P$ comes equipped with an automorphism $\phi$ of period $2^{k}$ (as in the present case, via conjugation) we would like to find a $\phi$ invariant normal chief series. We will call a representation of $C(k)$ over $\mathbf{Z}_{p}$ "homocyclic" if it decomposes into irreducible subrepresentations each having the same kernel.

LemMa 2. A p-group $P$ with an automorphism $\phi$ of period $2^{k}$ has a $\phi$ invariant normal chief series whose adjacent quotients $P_{i-1} / P_{l}$ are homocyclic representations of $C(k)$ with kernels $C\left(k_{i}\right)$, and $k_{i} \leq k_{i+1}$.

Proof. For any $p$-group, $P$, the characteristic subgroup $P^{\prime} P^{p}\left(P^{\prime}\right.$ is the commutator subgroup, $P^{p}$ is generated by all $p$ th powers) is called the Frattini subgroup, $\hat{P} . P / \hat{P}$ is elementary abelian and representatives in $P$ of generators of $P / \hat{P}$ will generate $P$. Moreover, $\hat{P}=e$ iff $P$ is elementary abelian (see [5; Ch. 5, Thm. 1.1]). 
Set $P_{0}=P$, consider the projection $\pi: P_{0} \rightarrow P_{0} / \hat{P}_{0}$ and suppose that the representation of $C(k)$ on $P_{0} / \hat{P}_{0}$ decomposes into $V_{1} \oplus \bar{V}_{1}$, where $V_{1}$ is the sum of all irreducible summands having the same, minimal kernel among the kernels appearing on $P_{0} / \hat{P}_{0}$, say $C\left(k_{1}\right)$. Now let $P_{1} \triangleleft P_{0}$ be $\pi^{-1}\left(\bar{V}_{1}\right)$. Then on $P_{0} / P_{1}, C(k)$ acts with kernel $C\left(k_{1}\right)$. Consider $P_{1} / \hat{P}_{1}$ and write $P_{1} / \hat{P}_{1}$ as $V_{2} \oplus \bar{V}_{2}$, where again $V_{2}$ is the sum of all irreducible summands with minimal kernel, say $C\left(k_{2}\right) . k_{2} \geq k_{1}$ because generators for $P_{1} / \hat{P}_{1}$ lift to generators for $P_{1}$ and $C\left(k_{1}\right)$ acts trivially on $P_{0}$ hence on $P_{1}$ by [5; Thm. 1.4]. Let $P_{2}=\pi^{-1}\left(\bar{V}_{2}\right)$ where $\pi: P_{1} \rightarrow P_{1} / \hat{P}_{1}$. This process can be continued until a $P_{j}$ is found such that $\hat{P}_{J}=e$. But then $P_{J}$ is elementary abelian and certainly $P_{j}$ can continue to be decomposed in this way. Thus we have a normal series

$$
e=P_{m} \triangleleft P_{m-1} \triangleleft \cdots \triangleleft P_{1} \triangleleft P_{0}=P
$$

such that $C(k)$ acts on $P_{i-1} / P_{i}$ with $C\left(k_{i}\right)$ and $k_{l} \leq k_{l+1}, i=1,2, \ldots, m$.

3. Special cases and the Main Theorem. If $G$ acts on a mod- $p$ homology sphere $X$, we wish to compare the degree, $\delta_{X^{P}}$, of a generator of $C(k)$ acting on $X$ with the degree, $\delta_{X^{P}}$, of the generator on $X^{P}$ (the induced action since $P \triangleleft G)$. The following lemma is central and is a modification of a key result of [8, compare Prop. 1.1].

Lemma 3. Suppose $G$ is a semidirect product of an elementary abelian p-group $P$ and a cyclic 2-group $C(k)$ such that the action of $C(k)$ on $P$ (by conjugation) has kernel $C(m)$ and is irreducible. If $G$ acts on a mod- $p$ homology $n$-sphere $X$ then the degrees $\delta_{X}$ and $\delta_{X^{p}}$ are related as follows:

$$
\delta_{X}=(-1)^{\varepsilon} \delta_{X^{P}} \quad \text { where } \varepsilon=\frac{n-n(P)}{2^{k-m}} .
$$

Proof. Proceeding exactly as in [8, loc. cit.], we consider the relative fibration $(X, Z) \rightarrow\left(X_{P}, Z_{P}\right) \stackrel{\pi}{\rightarrow} B P$, where $Z=X^{P} \sim_{p} S^{r}$. There is the spectral sequence of this relative fibering with $E_{2}$-term given by $E_{2}^{i, j}=$ $H^{i}(B P) \otimes H^{j}(X, Z)$ (coefficients in $Z_{p}$ ). If $d: E_{2}^{0, n} \rightarrow E_{2}^{n-r, r+1}$ (where $r=n(P))$ is the transgression then $d(x)=A \otimes \delta z$ where $x$ generates $H^{n}(X)$ and $z$ generates $H^{r}(Z)$. If rank $P$ is 1 then $A=t^{(n-r) / 2}$, where $t$ generates $H^{2}(B P)$. If rank $P>1$ then recall the Borel identity, $n-r=$ $\Sigma(n(H)-r)$, with sum on all corank 1 subgroups $H$ in $P$. Suppose there are exactly $s$ corank 1 subgroups $H_{1}, \ldots, H_{s}$ such that $n\left(H_{i}\right)-r>0$. Letting $r_{i}=n\left(H_{i}\right)$, there are elements $w_{1}, \ldots, w_{s} \in H^{2}(B P)$ and an $a \in$ $H^{0}(B P)$ such that $A=a w_{1}^{d_{1}} w_{2}^{d_{2}} \cdots w_{s}^{d_{s}}$ where $d_{l}=\left(r_{l}-r\right) / 2$ (see [6, Thm. 2]). 
Since $P$ is an irreducible representation of $C(k)$ (let $\alpha$ be a generator) with kernel $C(m), P$ has either dimension 1 (if either $k-m=1$ or if $k-m>1$ and $p \equiv 1(\bmod 4)$ with $k-m \leq l$, where $l$ is as defined in the proof of Lemma 1 (b) and depends only on $p$ ), or has dimension $2^{k-m-l}$ $\left(\right.$ resp. $\left.2^{k-m-l+1}\right)($ if $p \equiv 1(\bmod 4)$, resp. $p \equiv 3(\bmod 4))$.

Now, just as in [8], $C(k)$ acting by conjugation on $P$ determines an action of $C(k)$ on the fibration (and so on the spectral sequence) as follows. Define

$$
\phi: E G \times X \rightarrow E G \times X \quad \text { by } \phi(e, x)=\left(e \alpha, \alpha^{-1} x\right)
$$

where $\alpha$ generates $C(k)$. For $g \in P$, we have $\phi(g(e, x))=\psi(g) \phi(e, x)(\psi$ is the automorphism of $P$ defined by $\alpha^{-1} g \alpha=\psi(g)$ ). Thus we have an action on the fibration (since $E G \simeq E P$ ):

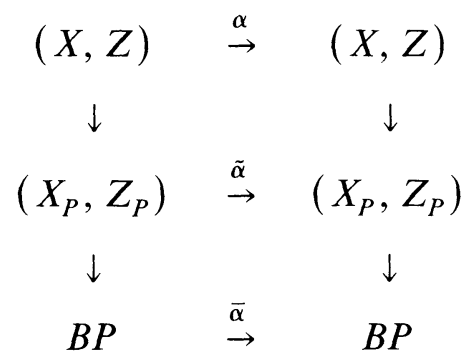

$\bar{\alpha}: B P \rightarrow B P$ is induced by $\psi: P \rightarrow P$. If $P$ has dimension $1, \bar{\alpha}^{*}(t)=\lambda t, \psi:$ $P \rightarrow P$ is multiplication by $\lambda$, a $2^{k-m-1}$ root of -1 and $t$ generates $H^{2}(B P)$. If the dimension of $P$ is larger than 1 , the action of $C(k)$ on the collection of subgroups $\left\{H_{1}, \ldots, H_{s}\right\}$ must be considered (and the corresponding action on $\left.w_{1}, \ldots, w_{s}\right)$. First of all, if $p \equiv 1(\bmod 4), \alpha^{2^{k-m-1}}$ acts on $P$ by multiplication on the basis elements by $\lambda$, a $2^{l-1}$ root of -1 and no smaller power of $\alpha$ leaves the $H_{l}$ invariant (smaller powers are represented by even dimensional irreducible subrepresentations). If $p \equiv 3$ ( $\bmod 4)$, since there are no roots of -1 in $\mathbf{Z}_{p}$, the smallest power of $\alpha$ leaving the $H_{l}$ invariant is $\alpha^{2^{k-m-1}}$ (this is just multiplication by -1 ). Therefore the members of $\left\{H_{1}, \ldots, H_{s}\right\}$ are permuted, each one in a orbit of size $2^{k-m-1}($ if $p \equiv 1(\bmod 4))$ or size $2^{k-m-1}($ if $p \equiv 3(\bmod 4))$. This observation has several consequences. If $H_{t}$ and $H_{J}$ are in the same orbit, $\left(n\left(H_{l}\right)-r\right) / 2=\left(n\left(H_{j}\right)-r\right) / 2$ and it follows from the Borel Identity that $2^{k-m-l}(p \equiv 1(\bmod 4))$ or $2^{k-m-1}(p \equiv 3(\bmod 4))$ divides $(n-r) / 2$. Now consider the class $a w_{1}^{d_{1}} \cdots w_{s}^{d_{s}}$. It follows from [6; Thm. 2; Lemma 3] that if $w_{i_{1}}, \ldots, w_{l_{2} k-m-1}(p \equiv 1(\bmod 4))$ or $w_{l_{1}}, \ldots, w_{l_{2 k-m-1}}(p \equiv 3(\bmod 4))$ are in the same orbit, the classes are permuted, say $w_{l_{j}} \rightarrow w_{l_{j+1}}$ and $w_{i_{2^{k-m-l}}} \rightarrow \lambda w_{i_{1}}\left(\lambda\right.$ a $2^{l-1}$ root of -1 and $\left.p \equiv 1(\bmod 4)\right)\left(\right.$ or $w_{l_{2 k-m-l}} \rightarrow-w_{i_{1}}$ 
if $p \equiv 3(\bmod 4))$. Under $\bar{\alpha}^{*}$ the class $a w_{1}^{d_{1}} \cdots w_{s}^{d_{s}}$ is sent to $\lambda^{\varepsilon} a w_{1}^{d_{1}} \cdots w_{s}^{d_{s}}$ (or $(-1)^{\varepsilon} a w_{1}^{d_{1}} \cdots w_{s}^{d_{s}}$ ) where $\varepsilon=(n-r) / 2^{k-m-l+1}$ (or $(n-r) / 2^{k-m}$ if $p \equiv 3(\bmod 4))$.

Consider now the commutative diagram (from the $E_{2}$-term):

$$
\begin{array}{ccc}
H^{n}(X, Z) & \stackrel{\alpha^{*}}{\rightarrow} & H^{n}(X, Z) \\
\downarrow d & & \downarrow d \\
H^{n-r}(B P) \otimes H^{r+1}(X, Z) & \stackrel{\alpha^{*} \otimes \alpha^{*}}{\rightarrow} & H^{n-r}(B P) \otimes H^{r+1}(X, Z) .
\end{array}
$$

We have:

$$
\begin{aligned}
d\left(\alpha^{*} x\right) & =\delta_{X}(A \otimes \delta z)=\left(\bar{\alpha}^{*} \otimes \alpha^{*}\right)(A \otimes \delta z) \\
& =\lambda^{\varepsilon} \delta_{X^{P}}(A \otimes \delta z) \quad\left(\text { or }(-1)^{\varepsilon} \delta_{X^{P}}(A \otimes \delta z)\right)
\end{aligned}
$$

where

$$
\varepsilon= \begin{cases}(n-r) / 2^{k-m-l+1} & \text { if } p \equiv 1(\bmod 4) \\ (n-r) / 2^{k-m} & \text { if } p \equiv 3(\bmod 4)\end{cases}
$$

Thus

$$
\delta_{X}=\lambda^{\varepsilon} \delta_{X^{P}} \quad\left(\text { or }(-1)^{\varepsilon} \delta_{X^{P}}\right) .
$$

Since each of $\delta_{X}, \delta_{X^{P}}$ is \pm 1 , it follows that if $k-m \leq l$,

$$
2^{k-m-1} \mid(n-r) / 2
$$

while if $k-m>l$,

$$
2^{l-1} \mid(n-r) / 2^{k-m-l+1}
$$

(all of this only when $p \equiv 1(\bmod 4))$.

Finally we have,

$$
\delta_{X}=(-1)^{\varepsilon} \delta_{X^{P}} \quad \text { where } \varepsilon=(n-r) / 2^{k-m} .
$$

This completes Lemma 3.

We can now prove an analogue of [8, Thm. 1.3]. Suppose $G$ is a semidirect product of a $p$-group $P$ and $C(k)$. Also, suppose that $G$ acts on a $\mathbf{Z}_{p}$-homology $n$-sphere $X$.

LEMMA 4. There is a sequence of subgroups $e=P_{m} \triangleleft P_{m-1} \triangleleft \cdots \triangleleft P_{1}$ $\triangleleft P_{0}=P$ and a corresponding sequence of non-negative integers $k_{1} \leq k_{2} \leq$ $\cdots \leq k_{m}$ such that if $\delta_{X}$ and $\delta_{X^{P}}$ denote, respectively, the degrees of $a$ 
generator $\alpha$ of $C(k)$ on $X, X^{P}$ then

$$
\delta_{X}=(-1)^{\varepsilon} \delta_{X^{P}}
$$

where

$$
\varepsilon=\sum_{i=1}^{m} \frac{n\left(P_{i}\right)-n\left(P_{t-1}\right)}{2^{k-k_{i}}}
$$

Proof. This now follows directly from Lemmas 2 and 3 applied to the $P_{l-1} / P_{i}$ action on $X^{P_{l}}$, where a normal series is obtained as in Lemma 2 and a refinement made so that adjacent quotients are irreducible.

The proof of the following is now clear.

THEOREM 1. If $G$ is a semidirect product as above, acting on a mod- $2 p$ homology n-sphere $X$, then $\chi\left(X^{G}\right)=\chi\left(S^{d}\right)$ where

$$
d \equiv n\left(\mathbf{Z}_{2^{k}}\right)-\left(\sum_{i=1}^{m} \frac{n\left(P_{i}\right)-n\left(P_{i-1}\right)}{2^{k-k_{i}}}\right)
$$

where the $P_{l}$ and $k_{i}$ are as in Lemma 4.

Proof. From a well-known result of Floyd $([4]), \chi\left(X^{G}\right)$ is the Lefschetz number of a generator of $\mathbf{Z}_{2^{k}}$ acting on $X^{P}$. One can easily verify that (from Lemma 4),

$$
\delta_{X^{P}}=(-1)^{n-n\left(\mathbf{Z}_{2 k}\right)+\varepsilon} .
$$

Since $n+n(P)$ is even,

$$
\chi\left(X^{G}\right)=1+(-1)^{n\left(\mathbf{Z}_{2 k}\right)-\varepsilon} .
$$

This completes the proof of Theorem 1 .

COROLlaRY. If $G$ and $X$ are as in Theorem 1 and, moreover, $G$ is a direct product then

$$
\chi\left(X^{G}\right)=\chi\left(X^{\mathbf{Z}_{2^{k}}}\right) .
$$

Proof. The reader may check that in this case the sum term appearing in the conclusion is $0 \bmod 2$ (this is easy to see via Lemma 3). Note that this corollary is also easily obtained from a well-known result of Floyd (see [1; Ch. III, Th. 4.4.]).

REMARK. Suppose $G$ is an extension of an elementary abelian $p$-group $P$ by a cyclic 2-group $\mathbf{Z}_{2^{k}}, P \gg G \rightarrow \mathbf{Z}_{2^{k}}$ and $\psi: \mathbf{Z}_{2^{k}} \rightarrow \operatorname{Aut}(P)$ has kernel 
$\mathbf{Z}_{2^{m}}$. If $V$ is a real representation of $G$ then we have:

$$
\operatorname{dim} V^{G} \equiv \operatorname{dim} V^{\mathbf{Z}_{2 k}}-\left(\frac{\operatorname{dim} V-\operatorname{dim} V^{P}}{2^{k-m}}\right) \quad(\bmod 2) .
$$

This can be verified by considering the real irreducible representations of $G$, which originate from complex irreducible representation which in turn are induced up from complex irreducible representations of the subgroup $P \times \mathbf{Z}_{2^{m}}$. If those complex irreducible representations of $G$, for which both $P$ and $\mathbf{Z}_{2^{m}}$ act nontrivially, are compared with those for which $P$ acts nontrivially but $\mathbf{Z}_{2^{m}}$ acts trivially, the congruence above can be derived. It should also be noted that if $m=0$ then the above congruence is actually an equality (for more information see [7; Chapters 7, 8 and 13]).

\section{REFERENCES}

[1] A. Borel, (et al), Amer. Math. Studies (Princeton Univ. Press), 1960, \# 46.

[2] R. Dotzel and G. Hamrick, p-group actions on homology spheres, Invent. Math., 62 (1981), 437-442.

[3] K. H. Dovermann and Ted Petrie, Artin relations for smooth representations, Proc. Ntl. Acad. Sci. USA, Vol. 77, No. 10, (1980), 5620-5621.

[4] E. Floyd, On related periodic maps, Amer. J. Math., 74 (1952), 547-554.

[5] D. Gorenstein, Finite Groups, Harper, 1968.

[6] W.-Y. Hsiang, Generalizations of a theorem of Borel, Proceedings of the University of Georgia Topology of Manifolds Institute (1969), 274-290.

[7] J.-P. Serre, Linear Representations of Finite Groups, Springer-Verlag, GTM 42.

[8] E. Straume, Dihedral transformation groups of homology spheres, J. Pure and Applied Algebra, 21 (1981), 51-74.

Received August 4, 1982 and in revised form December 3, 1982. This research supported in part by Summer Research Fellowship (University of Missouri-St. Louis).

UNIVERSITY OF MISSOURI-ST. LOUIS

ST. LouIs, MO 63121 



\section{PACIFIC JOURNAL OF MATHEMATICS EDITORS}

\author{
DONALD BABBITT (Managing Editor) \\ University of California \\ Los Angeles, CA 90024 \\ J. DugundJI \\ University of Southern California \\ Los Angeles, CA 90089-1113 \\ R. FINN \\ Stanford University \\ Stanford, CA 94305 \\ HERMANN FLASChKa \\ University of Arizona \\ Tucson, AZ 85721
}

C. C. MOORE

University of California

Berkeley, CA 94720

Arthur Ogus

University of California

Berkeley, CA 94720

Hugo RossI

University of Utah

Salt Lake City, UT 84112

H. SAMELSON

Stanford University

Stanford, CA 94305

ASSOCIATE EDITORS
R. ARENS
E. F. BECKENBACH
B. H. NeUmanN
F. WOLF
K. YosHIDA (1906-1982)

\section{SUPPORTING INSTITUTIONS}
UNIVERSITY OF ARIZONA
UNIVERSITY OF BRITISH COLUMBIA
CALIFORNIA INSTITUTE OF TECHNOLOGY
UNIVERSITY OF CALIFORNIA
MONTANA STATE UNIVERSITY
UNIVERSITY OF NEVADA, RENO
NEW MEXICO STATE UNIVERSITY
OREGON STATE UNIVERSITY

\author{
UNIVERSITY OF OREGON \\ UNIVERSITY OF SOUTHERN CALIFORNIA \\ STANFORD UNIVERSITY \\ UNIVERSITY OF HAWAII \\ UNIVERSITY OF TOKYO \\ UNIVERSITY OF UTAH \\ WASHINGTON STATE UNIVERSITY \\ UNIVERSITY OF WASHINGTON
}

The Supporting Institutions listed above contribute to the cost of publication of this Journal, but they are not owners or publishers and have no responsibility for its content or policies.

Mathematical papers intended for publication in the Pacific Journal of Mathematics should be in typed form or offset-reproduced (not dittoed), double spaced with large margins. Please do not use built up fractions in the text of the manuscript. However, you may use them in the displayed equations. Underline Greek letters in red, German in green, and script in blue. The first paragraph must be capable of being used separately as a synopsis of the entire paper. In particular it should contain no bibliographic references. Please propose a heading for the odd numbered pages of less than 35 characters. Manuscripts, in triplicate, may be sent to any one of the editors. Please classify according to the scheme of Math. Reviews, Index to Vol. 39. Supply name and address of author to whom proofs should be sent. All other communications should be addressed to the managing editor, or Elaine Barth, University of California, Los Angeles, California 90024.

There are page-charges associated with articles appearing in the Pacific Journal of Mathematics. These charges are expected to be paid by the author's University, Government Agency or Company. If the author or authors do not have access to such Institutional support these charges are waived. Single authors will receive 50 free reprints; joint authors will receive a total of 100 free reprints. Additional copies may be obtained at cost in multiples of 50 .

The Pacific Journal of Mathematics is issued monthly as of January 1966. Regular subscription rate: $\$ 190.00$ a year (5 Vols., 10 issues). Special rate: $\$ 66.00$ a year to individual members of supporting institutions.

Subscriptions, orders for numbers issued in the last three calendar years, and changes of address should be sent to Pacific Journal of Mathematics, P.O. Box 969, Carmel Valley, CA 93924, U.S.A. Old back numbers obtainable from Kraus Periodicals Co., Route 100, Millwood, NY 10546.

The Pacific Journal of Mathematics at P.O. Box 969, Carmel Valley, CA 93924 (ISSN 0030-8730) publishes 5 volumes per year. Application to mail at Second-class postage rates is pending at Carmel Valley, California, and additional mailing offices. Postmaster: Send address changes to Pacific Journal of Mathematics, P.O. Box 969, Carmel Valley, CA 93924.

PUBLISHED BY PACIFIC JOURNAL OF MATHEMATICS, A NON-PROFIT CORPORATION

Copyright $\odot 1984$ by Pacific Journal of Mathematics 


\section{Pacific Journal of Mathematics}

Vol. 114, No. $2 \quad$ June, 1984

William Allen Adkins, A Harnack estimate for real normal surface

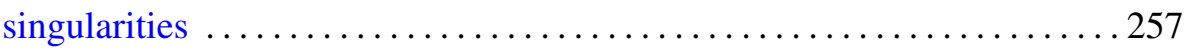

George E. Andrews, Multiple series Rogers-Ramanujan type identities . . . . 267

Didier Arnal, $*$ products and representations of nilpotent groups . . . . . . 285

David Cox and Walter Raymond Parry, Representations associated with

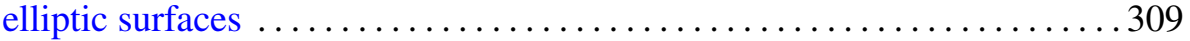

Joanne Marie Dombrowski, Tridiagonal matrix representations of cyclic

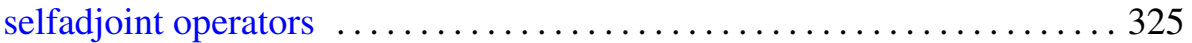

Ronald Dotzel, An Artin relation $(\bmod 2)$ for finite group actions on

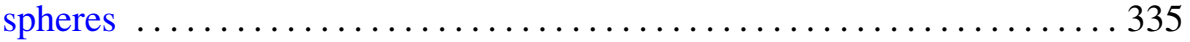

Leo Egghe, Convergence of adapted sequences of Pettis-integrable

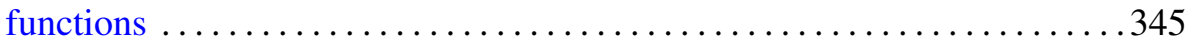

Rebecca A. Herb, Characters of induced representations and weighted

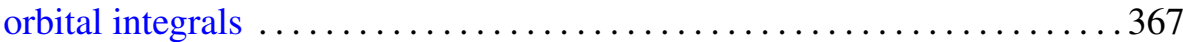

Steven M. Kahn, Cobordism obstructions to fibering manifolds over

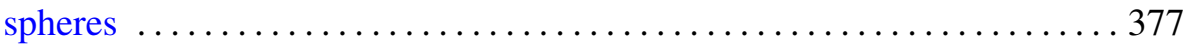

Robert D. Little, Projective space as a branched covering of the sphere with orientable branch set

Claude Schochet, Topological methods for $C^{*}$-algebras. III. Axiomatic

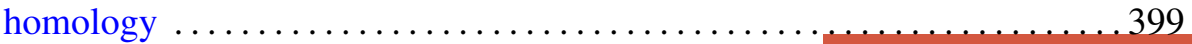

Claude Schochet, Topological methods for $C^{*}$-algebras. IV. $\bmod p$ homology

James M. Stormes, On the $K O$-orientability of complex projective varieties

Josephine Anne Ward, Characterization of homogeneous spaces and their norms 